УДК 004.8

\title{
МЕТОД ОЦЕНИВАНИЯ ПОСЛЕДОВАТЕЛЬНО ОТСУТСТВУЮЩИХ ДАННЫХ СЕНСОРОВ С ИСПОЛЬЗОВАНИЕМ ГЛУБОКОЙ НЕЙРОННОЙ СЕТИ*
}

\author{
ФЭН ЛЮ, ХУЙлинь ЛИ, ЧжУН ян
}

Уайчжунский сельскохозяйственный университет, Китай, Ухань, Хубэй

\begin{abstract}
Аннотация - Явление отсутствующих измерений достаточно распространено в беспроводных сенсорных сетях WSN (wireless sensor networks). Оно оказывает существенное влияние на удобство использования, стабильность и эффективность приложений на основе WSN. Существует множество методов оценивания отсутствующих измерений. Однако точное и эффективное последовательное оценивание отсутствующих измерений остается сложной задачей. Чтобы решить эту проблему, предложен новый метод, названный последовательным оцениванием сенсорных измерений на основе глубокой нейронной сети CSDNN (consecutive sensor data deep neural network). В этом методе, во-первых, анализируются коэффициенты корреляции между различными типами измерений и выбирается определенное количество ближайших соседей из целевого сенсорного узла. Во-вторых, для оценки определенного типа измерений используются различные типы измерений с сильной корреляцией и измерениями одного и того же типа от вышеупомянутых ближайших соседей. Эти измерения рассматриваются как входные данные для глубокой нейронной сети DNN (deep neural network). B-третьих, построена модель DNN, обсуждается оптимизированная структура DNN в случае отсутствующих измерений и проверяется точность CSDNN для различных типов измерений окружающей среды. Согласно полученным результатам, CSDNN позволяет точно оценивать последовательно отсутствующие измерения.
\end{abstract}

Ключевые слова: беспроводная сенсорная сеть; отсутствующие данные сенсоров; оценивание отсутствующих измерений; глубокая нейронная сеть

\section{1. ВСТУПЛЕНИЕ}

Беспроводные сенсорные сети WSN (wireless sensor networks) получили широкое распространение в последнее время. Поэтому многие связанные с ними проблемы стали актуальными темами исследований. Эти проблемы включают проблемы систем позиционирования [1], методы энергосбережения [2], кодирование данных и оптимальный путь передачи
[3], качество обслуживания [4], отслеживание движущихся радиоисточников [5] и т.д.

Приложения, основанные на WSN, собирают множество измерений с внешних сенсоров. Однако неблагоприятные условия среды или ограниченная энергия сенсора приводят к явлениям отсутствующих измерений. Другой вероятной причиной последовательного отсутствия измерений является «истощение» сен-

* Проект профинансирован фондами фундаментальных исследований (Fundamental Research Funds) для центральных университетов (программа № 2662017JC028) и провинциальным научным фондом Китая в провинции Хубэй (Hubei Provincial Natural Science Foundation of China), грант № 2015CFB437. 


\section{БИБЛИОГРАФИЧЕСКИЙ СПИСОК}

1. Belakbir, A.; Amghar, M.; Sbiti, N. "Sensor data fusion for an indoor and outdoor localization," Radioelectron. Commun. Syst., Vol. 57, No. 4, P. 149-158, 2014. DOI: $10.3103 / \mathrm{S} 0735272714040013$.

2. Kaur, J.; Randhawa, S.; Jain, S. “A novel energy efficient cluster head selection method for wireless sensor networks," Int. J. Wireless Microwave Technol., Vol. 8, No. 2, P. 37-51, 2018. DOI: 10.5815/ijwmt.2018.02.04.

3. Chen, J.; Yatskiv, V. V.; Sachenko, A. A.; Su, J. "Wireless sensor networks based on modular arithmetic," Radioelectron. Commun. Syst., Vol. 60, No. 5, P. 215-224, 2017. DOI: $10.3103 / \mathrm{S} 073527271705003 \mathrm{X}$.

4. Qushtom, H.; Rabaya, K. "Enhancing the QoS of IoT networks with lightweight security protocol using Contiki OS," Int. J. Computer Network Inf. Security, Vol. 9, No. 11, P. 27-35, 2017. DOI: 10.5815/ijcnis.2017. $\underline{11.03 .}$.

5. Tovkach, I. O.; Zhuk, S. Y. “Adaptive filtration of radio source movement parameters with complex use of sensor network data based on TDOA and RSS methods," Radioelectron. Commun. Syst., Vol. 60, No. 12, P. 528-537, 2017. DOI: 10.3103/S0735272717120020. 
6. Mishra, N.; Soni, H. K.; Sharma, S.; Upadhyay, A. $\mathrm{K}$. "Development and analysis of artificial neural network models for rainfall prediction by using time-series data," Int. J. Intelligent Systems Appl., Vol. 1, P. 16-23, 2018. DOI: $10.5815 /$ ijisa.2018.01.03.

7. Zhang, Hongguo; Yang, Liang. "An improved algorithm for missing data in wireless sensor networks," Proc. of Int. Conf. on Software Intelligence Technologies and Applications \& International Conf. on Frontiers of Internet of Things 2014, 4-6 Dec. 2014, Hsinchu, Taiwan. IEEE, 2014, P. 346-350. DOI: 10.1049/cp.2014.1587.

8. Pan, L.; Gao, Huijun; Li, J.; Gao, Hong; Guo, Xintong. "CIAM: An adaptive 2-in-1 missing data estimation algorithm in wireless sensor networks," Proc. of 2013 19th IEEE Int. Conf. on Networks, ICON, 10-13 Dec. 2013, Singapore. IEEE, 2013, pp. 1-6. DOI: 10.1109/ ICON.2013.6781986.

9. Alippi, C.; Ntalampiras, S.; Roveri, M. "Model ensemble for an effective on-line reconstruction of missing data in sensor networks," Proc. of 2013 Int. Joint Conf. on Neural Networks, IJCNN, 4-9 Aug. 2013, Dallas, TX, USA. IEEE, 2013, pp. 1-6. DOI: 10.1109/IJCNN.2013. 6706761.

10. Satter, A.; Ibtehaz, N. “A regression based sensor data prediction technique to analyze data trustworthiness in cyber-physical system," Int. J. Inf. Eng. Electron. Business, Vol. 3, P. 15-22, 2018. DOI: 10.5815/ijieeb. 2018.03.03.

11. Gruenwald, L.; Yang, H.; Sadik, M. S.; Shukla, R. "Using data mining to handle missing data in multi-hop sensor network applications," Proc. of Ninth ACM Int.
Workshop on Data Engineering for Wireless and Mobile Access, 6 Jun. 2010, Indianapolis, Indiana. ACM, 2010. DOI: $10.1145 / 1850822.1850825$.

12. Niu, K.; Zhao, F.; Qiao, X. "A missing data imputation algorithm in wireless sensor network based on minimized similarity distortion," Proc. of 2013 Sixth Int. Symp. on Computational Intelligence and Design, 28-29 Oct. 2013, Hangzhou, China. IEEE, 2013, pp. 235-238. DOI: 10.1109/ISCID.2013.172.

13. Guo, D.; Qu, X.; Huang, L.; Yao, Y.; Liu, Z.; Sun, M.-T. "Sparsity-based online missing sensor data recovery," Proc. of 2012 IEEE Int. Symp. on Circuits and Systems, 20-23 May 2012, Seoul, South Korea. IEEE, 2012, pp. 918-921. DOI: 10.1109/ISCAS.2012.6272193.

14. Fekade, B.; Maksymyuk, T.; Kyryk, M.; Jo, M. "Probabilistic recovery of incomplete sensed data in IoT," IEEE IoT J., Vol. PP, No. 99, p. 1-1, 2017. DOI: 10.1109/JIOT.2017.2730360.

15. Xie, K.; Ning, X.; Wang, X.; Xie, D.; Cao, J.; Xie, G.; Wen, J. "Recover corrupted data in sensor networks: A matrix completion solution," IEEE Trans. Mobile Computing, Vol. 16, No. 5, P. 1434-1448, 2017. DOI: 10.1109/TMC.2016.2595569.

16. Anagnostopoulos, C.; Triantafillou, P. "Scaling out big data missing value imputations: pythia vs. godzilla," Proc. of 20th ACM SIGKDD Int. Conf. on Knowledge Discovery and Data Mining, 24-27 Aug. 2014, New York, USA. ACM, 2014, DOI: 10.1145/2623330.2623615.

17. Schmidhuber, J. "Deep learning in neural networks: An overview," Neural Networks, Vol. 61, pp. 85-117, Jan. 2015. DOI: 10.1016/j.neunet.2014.09.003. 